CERN-TH/2000-096

hep-th/0003226

March, 2000

\title{
Non-BPS D-brane Solutions in Six Dimensional Orbifolds
}

\author{
Yolanda Lozano \\ Theory Division, CERN \\ 1211 Genève 23, Switzerland \\ yolanda.lozano@cern.ch
}

\begin{abstract}
Starting with the non-BPS D0-brane solution of IIB/ $(-1)^{F_{L}} I_{4}$ constructed recently by Eyras and Panda we construct via T-duality the non-BPS D2-brane and D1-brane solutions of IIB/ $/(-1)^{F_{L}} I_{4}$ and IIA $/(-1)^{F_{L}} I_{4}$ predicted by Sen. The D2-brane couples magnetically to the vector field of the NS5B-brane living in the twisted sector of the Type IIB orbifold, whereas the D1-brane couples (electrically and magnetically) to the self-dual 2-form potential of the NS5A-brane that is present in the twisted sector of the Type IIA orbifold construction. Finally we discuss the eleven dimensional interpretation of these branes as originating from a non-BPS M1-brane solution of M-theory orientifolded by $\Omega_{\rho} I_{5}$.
\end{abstract}

PACS: 11.25.-w; 11.27.+d

Keywords: Branes; Duality; Supergravity 


\section{Introduction and Summary}

The study of brane-antibrane systems in string and M-theory has brought a new perspective onto the understanding of non-perturbative extended objects (see [1]-[4], and references therein). BPS branes arise as by-products when the tachyonic mode of the open strings connecting the brane and the antibrane condenses in a vortex-like configuration. Also, one can deduce in this framework the presence of new non-BPS extended objects when the tachyon condenses, instead, in a kink-like configuration. These objects have subsequently been shown to play a key role in testing string dualities beyond the BPS level. In Type II and M-theory they are however unstable, because the open strings ending on them contain real tachyonic excitations, but the instability can be cured when the theory is projected out by a certain symmetry that removes the tachyons from the spectrum. This happens in particular for some non-BPS branes in Type I and in certain orbifold/orientifold constructions of Type II and M-theory.

An interesting and still open problem is the construction of the supergravity solutions corresponding to these non-BPS branes. In the Type II theories given that they are unstable one does not expect to find stable classical solutions of the supergravity that could be associated to these branes. Only in some cases it has been possible to construct classical solutions, like the one corresponding to a Kaluza-Klein monopole-antimonopole pair in M-theory, that is stabilized by suspending the system in an external magnetic field (see [5] and [6, 7, 8, 9, 10] for related work), or the solution of the, unstable, nonBPS D-instanton of Type IIA constructed in [11]. For the stable non-BPS branes one however expects to find solutions of the supergravity equations of motion that describe these branes in the strong coupling regime.

Recently, using the boundary state formalism, Eyras and Panda [12 found the asymptotic behavior of the solution corresponding to the non-BPS D0brane of the Type IIB theory orbifolded by $(-1)^{F_{L}} I_{4}$ [13, 14]. This theory is S-dual to Type IIB orientifolded by $\Omega I_{4}, \Omega$ being the worldsheet parity reversal operation. The twisted sector of this orientifold construction of Type IIB consists, in the compact case, on 16 O5 orientifold fixed planes together with a D5-brane on top of each plane, each D5-O5 system carrying an $\mathrm{SO}(2)$ vector potential. This theory contains massive non-BPS states in the perturbative spectrum arising from open strings stretched between a D5-brane and its image [15]. These states are stable, since they are the lightest states charged under the $\mathrm{SO}(2)$ gauge field of the twisted sector, and correspond in

\footnotetext{
${ }^{1}$ Here $F_{L}$ denotes the left-moving spacetime fermion number, and $I_{4}: x^{i} \rightarrow-x^{i} ; i=$ $1, \ldots 4$.
} 
the strong coupling limit to the non-BPS D0-branes of IIB/ $(-1)^{F_{L}} I_{4}$ [13, 14]. These non-BPS D0-branes are also charged with respect to the $\mathrm{SO}(2)$ vector field of the, S-dual, twisted sector, and this renders them stable. Moreover, for a critical value of the radii of the compact orbifold a pair of branes satisfies a no-force condition at least when the distance is larger than the string scale [16, 17]2. This opens the possibility of constructing solutions corresponding to a large number of parallel non-BPS D0-branes, which could correctly describe the weak coupling regime of the theory. More importantly for our discussion, it allows the possibility of constructing infinite arrays of non-BPS D0-branes, from where one can derive other non-BPS D-brane solutions via T-duality transformations.

In this paper we concentrate on this and other stable non-BPS branes, that occur in the six dimensional orbifold/orientifold constructions obtained by projecting out the Type IIB theory by $\Omega I_{4}$ and duality-related operations. In particular, we focus on the Type IIB and Type IIA theories divided out by $(-1)^{F_{L}} I_{4}$. The $(-1)^{F_{L}}$ operation is identified in the strong coupling limit as the transformation reversing the orientation of open D-strings (D2-branes) in the Type IIB (Type IIA) theory, as implied by its connexion via S-duality with the $\Omega$ symmetry of Type IIB [19] (in Type IIA a further T-duality transformation is required). Therefore, the twisted sector of Type IIB/ $(-1)^{F_{L}} I_{4}$ can be described non-perturbatively, in the compact case, as 16 O5-NS5B systems [20, 21], and that of Type IIA/ $(-1)^{F_{L}} I_{4}$ as the same number of O5NS5A systems [20]. An O5-plane with a NS5B-brane on top of it contains an $\mathrm{SO}(2)$ gauge field associated to open D-strings stretched between the NS5brane and its image, and the non-BPS D0-branes are charged with respect to this twisted field. In the Type IIA theory, the O5-NS5A system contains a self-dual 2-form field associated to open D2-branes stretched between a NS5A-brane and its image, and therefore the twisted sector contributes with $\mathrm{SO}(2)$ self-dual 2-form potentials $\mathrm{s}^{\mathrm{s}}$.

In [1] Sen conjectured that together with the non-BPS D0-brane, coupled electrically to the $\mathrm{SO}(2)$ vector field of the NS5B-O5 system, there is a nonBPS D2-brane, placed on the orbifold plane, that couples magnetically to the same vector field, and arises from open D3-branes stretched between a NS5B-brane and its mirror. Also, T-duality predicts a non-BPS D1-brane in IIA/ $(-1)^{F_{L}} I_{4}$ located on the orbifold plane, which should couple, electrically

\footnotetext{
${ }^{2}$ In [18] it is shown that for coincident branes a vacuum configuration in which the branes attract each other seems to be more favourable even at the critical radius. As mentioned in that reference this result is however challenged by the fact that the expectation value for the tachyon fields is beyond the range of validity of the approximation. It would be very interesting to clarify this point further.

${ }^{3}$ See [20] and [22, 23] for a more detailed description of the twisted sector.
} 
and magnetically, to the self-dual 2-form potential of the twisted sector, and arise from open D2-branes stretched between a NS5A-brane and its mirror.

In this note we construct these D-brane solutions. We also show that there is a non-BPS M1-brane solution of M-theory orientifolded by $\Omega_{\rho} I_{5}$ [22, 23], where $\Omega_{\rho}$ reverses the orientation of the M2-brane, to which all these branes are related by reduction and dualities. This provides a unifying picture within M-theory of the stable non-BPS branes that occur in the six dimensional orbifold/orientifold constructions related to $\Omega_{\rho} I_{5}$.

M-theory on a 5 -torus orientifolded by $\Omega_{\rho} I_{5}$ contains a twisted sector that can be identified as 16 O5-M5 systems, together with other 16 O5 orientifold fixed planes which do not contribute any twisted states [23]. This theory contains non-BPS M1-branes that arise from open M2-branes stretched between an M5-brane and its mirror [15. They couple, electrically and magnetically, to the self-dual 2-form potential living in the M5-brane, what makes them stable. In reducing to the Type IIA theory one can consider two possibilities:

1. Reduce along a worldvolume direction of the M5-O5 system. In this case one obtains Type IIA orientifolded by $\Omega I_{5}$, whose twisted sector states arise from 16 D4-O4 systems. This theory contains perturbative massive non-BPS states, which can be interpreted in M-theory as nonBPS M1-branes wrapped on the eleventh direction [15], as well as nonperturbative non-BPS strings coming from open D2-branes stretched between a D4-brane and its mirror, which correspond to unwrapped M1-branes in M-theory [1]. T-duality along one of the orbifolded directions gives then rise to the Type IIB theory orientifolded by $\Omega I_{4}$, whose twisted sector is described by 16 D5-O5 systems. This theory contains perturbative non-BPS particle states and non-perturbative non-BPS 2-branes, connected by T-duality with the non-BPS objects of IIA.

2. Reduce M-theory $/ \Omega_{\rho} I_{5}$ along one of the orbifolded directions. In this case one obtains Type IIA projected out by $(-1)^{F_{L}} I_{4}$, with a twisted sector consisting of 16 NS5-O5 systems. The non-BPS M1-brane gives rise to a non-BPS D1-brane in IIA that couples (electrically and magnetically) to the self-dual 2-form potential living in the worldvolume of the NS5A-O5. Now T-duality along a worldvolume direction of the NS5A-O5 maps the theory onto Type IIB divided by $(-1)^{F_{L}} I_{4}$, with a twisted sector identified as 16 NS5-O5 systems. Non-BPS D0-branes are coupled electrically to the $\mathrm{SO}(2)$ vector field of the twisted sector, and non-BPS D2-branes magnetically. These branes are related to the non-BPS D1-brane by T-duality.

Consistently with the whole duality picture [24], the two theories that are ob- 
tained by either reducing along an M5-brane direction and then T-dualizing along a transverse direction, or viceversa, are related by S-duality. In particular one obtains IIB $/ \Omega I_{4}$ and IIB $/(-1)^{F_{L}} I_{4}$ respectively. We also see that the non-BPS M1-brane of M-theory $/ \Omega_{\rho} I_{5}$ is the eleven dimensional origin of the non-BPS branes that can be defined in the Type II orbifolds/orientifolds obtained by reduction.

\section{The non-BPS D0-brane solution of [12]}

The asymptotic behavior of the solution corresponding to the non-BPS D0brane of Type IIB orbifolded by $(-1)^{F_{L}} I_{4}$ has been derived in 12 using the boundary state formalism. In this formalism one can compute the long distance behavior of the massless fields generated by the D-brane and predict in this manner the asymptotic form of the corresponding classical solution [25]. A pair of non-BPS D0-branes satisfies a no-force condition when the orbifold is compactified to a particular critical value of the radii [16, 17]. When this happens it is possible to construct periodic infinite arrays of nonBPS D0-branes and compute T-dual solutions, which is what we shall be doing in the next sections. Note that both the no-force condition and the validity of the classical solution and the T-duality rules hold for distances larger than the string scale.

The asymptotic form of the solution of [12], corresponding to a D0-brane situated at one of the fixed points of the orbifold, reads, in string framet:

$$
\begin{aligned}
d s_{D 0}^{2}= & -\left(1-\frac{1}{3} \frac{\kappa_{6} T_{0}}{2 \pi^{2} \Omega_{4}} \frac{1}{|y|^{3}}+\ldots\right) d t^{2}+ \\
& +\left(1+\frac{1}{3} \frac{\kappa_{6} T_{0}}{2 \pi^{2} \Omega_{4}} \frac{1}{|y|^{3}}+\ldots\right)\left(\delta_{m n} d y^{m} d y^{n}+\right. \\
& \left.+\delta_{i j} d x^{i} d x^{j}\right) ; m, n=1, \ldots 5 ; i, j=1, \ldots 4 \\
e^{\phi}=1 & +\frac{1}{2} \frac{\kappa_{6} T_{0}}{2 \pi^{2} \Omega_{4}} \frac{1}{|y|^{3}}+\ldots, \\
C_{0}^{(1)}= & -\frac{1}{3} \frac{\kappa_{6} Q_{0}}{\sqrt{2} \Omega_{4}} \frac{1}{|y|^{3}}+\ldots
\end{aligned}
$$

\footnotetext{
${ }^{4}$ In [12] a somewhat more general solution depending on a free parameter $a$ is given, derived by impossing the no-force condition of a pair of branes at the critical radii as a constraint for the background fields. Here we have chosen to work with the strictly linearized solution, though the same kind of generalization can be done for our solutions.
} 
Here we have taken $\alpha^{\prime}=1$ but otherwise the notation is that in [12]. Namely, $\kappa_{D}^{2}=8 \pi G_{D}, \kappa_{D-d}^{2}=\kappa_{D}^{2} / V_{d}$, with $V_{d}$ the volume of the $d$ dimensional space, $\Omega_{4}$ is the area of a unit sphere surrounding the D0-brane, $T_{0}$ is the tension of the brane, $Q_{0}$ its charge $y^{m}, m=1, \ldots 5$ the longitudinal directions along the NS5B-O5 worldvolume, and $x^{i}, i=1, \ldots 4$, the transverse, orbifolded directions. The critical value of the radii: $R_{c}=1 / \sqrt{2}$, has already been substituted in the solution. $C^{(1)}$ is the vector potential coming from the twisted sector, under which the D0-brane is charged.

\section{The non-BPS D1-brane of IIA/(-1 $)^{F_{L}} I_{4}$}

Considering a periodic infinite array of non-BPS D0-branes along the $y^{5}$ direction we can construct via T-duality a non-BPS D1-brane solution in the Type IIA theory projected out by $(-1)^{F_{L}} I_{4}$. This brane is situated at one of the fixed points of the orbifold with its worldsheet extended along the non-compact spacetime. We find:

$$
\begin{aligned}
d s_{D 1}^{2}= & \left(1-\frac{1}{2} \frac{\kappa_{6} T_{1}}{2 \pi^{2} \Omega_{3}} \frac{1}{|y|^{2}}+\ldots\right)\left(-d t^{2}+d \sigma^{2}\right)+ \\
& +\left(1+\frac{1}{2} \frac{\kappa_{6} T_{1}}{2 \pi^{2} \Omega_{3}} \frac{1}{|y|^{2}}+\ldots\right)\left(\delta_{m n} d y^{m} d y^{n}+\delta_{i j} d x^{i} d x^{j}\right) ; \\
& m, n=1, \ldots 4 ; i, j=1, \ldots 4, \\
e^{\phi}= & 1+\frac{1}{2} \frac{\kappa_{6} T_{1}}{2 \pi^{2} \Omega_{3}} \frac{1}{|y|^{2}}+\ldots, \\
C_{0 \sigma}^{(2)}= & \frac{1}{2} \frac{\kappa_{6} Q_{1}}{\sqrt{2} \Omega_{3}} \frac{1}{|y|^{2}}+\ldots
\end{aligned}
$$

Here $T_{1}$ is the tension of the brane, $Q_{1}$ its charge and $\Omega_{3}$ the area of the unit 3-sphere surrounding the string. The twisted sector consists on a 2-form potential, under which the D1-brane is charged. T-duality implies that this field is to be interpreted as the 2-form potential living in a NS5A-O5 system.

This D1-brane solution can be interpreted as a ten dimensional 1-brane located at the origin of the four-dimensional compact space. Considering first a single compactified direction, a 1-brane sitting at the origin of the $S^{1}$ can

\footnotetext{
${ }^{5}$ Impossing open-closed string consistency for boundary states, $T_{0}$ and $Q_{0}$ are fixed to: $T_{0}=8 \pi^{7 / 2}, Q_{0}=8 \sqrt{2} \pi^{3 / 2}$, (see [12]).
} 
be seen from the point of view of the covering space of the $S^{1}$ as an equally spaced array of D1-branes in the $S^{1}$ direction. If $\vec{x}$ denotes a vector in the full eight dimensional transverse space, we can then approximate $1 /|x|^{6}$ by a sum $\sum_{n \in Z} 1 /\left(r^{2}+\left(x^{4}-2 \pi n R_{4}\right)^{2}\right)^{3}$, with $r^{2}=\sum_{m=1}^{4} y^{m} y_{m}+\sum_{i=1}^{3} x^{i} x_{i}$, and, assuming that the size of the compact direction is smaller than the distance in the non-compact space, we can further approximate the sum by an integral. Repeating this process for the four compact directions we can finally write (see for instance 26] for more details):

$$
\frac{1}{|y|^{2}} \approx \frac{1}{|x|^{6}} \Pi_{i=1}^{4}\left(2 \pi R_{i}\right)\left(I_{1} I_{2} I_{3} I_{4}\right)^{-1}
$$

where $R_{i}$ are the radii of the compactified orbifold and $I_{n} \equiv \int_{0}^{\pi} d \theta \sin ^{n} \theta$. Substituting back in the expression for the D1-brane solution in the compact orbifold we can then obtain the corresponding solution in the uncompactified case:

$$
\begin{aligned}
d s_{D 1}^{2}= & \left(1-\frac{1}{6} \frac{\kappa_{10} T_{1}}{\Omega_{7}} \frac{1}{|x|^{6}}+\ldots\right)\left(-d t^{2}+d \sigma^{2}\right)+ \\
& +\left(1+\frac{1}{6} \frac{\kappa_{10} T_{1}}{\Omega_{7}} \frac{1}{|x|^{6}}+\ldots\right)\left(\delta_{m n} d y^{m} d y^{n}+\delta_{i j} d x^{i} d x^{j}\right) ; \\
& m, n=1, \ldots 4 ; i, j=1, \ldots 4, \\
e^{\phi}= & 1+\frac{1}{6} \frac{\kappa_{10} T_{1}}{\Omega_{7}} \frac{1}{|x|^{6}}+\ldots, \\
C_{0 \sigma}^{(2)}= & \frac{1}{2} \frac{\kappa_{6} Q_{1}}{\sqrt{2} \Omega_{3}} \frac{1}{|y|^{2}}+\ldots .
\end{aligned}
$$

Now $\Omega_{7}$ is the area of the unit 7 -sphere surrounding the string. The expression for the 2-form potential is the same as in the compactified case since it lives in the twisted sector. In [12] it is shown that for the non-BPS D0-brane this kind of approach relating the uncompactified and the compactified solutions gives the same answer than the boundary state analysis of the uncompactified orbifold. This should be the case also for the D1-brane.

It is straightforward to check that this D1-brane solution solves the equations of motion derived from an action $S_{\text {untwisted }}+S_{\text {twisted }}$, where:

$$
S_{\text {untwisted }}=\frac{1}{2 \kappa_{10}^{2}} \int d^{10} x e^{-2 \phi} \sqrt{|\operatorname{det} g|}\left(R+4(\partial \phi)^{2}\right),
$$

\footnotetext{
${ }^{6}$ The choice of this function will be clear below.
} 
and the action corresponding to the twisted sector is proportional to the worldvolume effective action associated to a pair NS5A-O5. This reads, in string frame and to first order in $\alpha^{\prime}$ :

$$
\begin{aligned}
S_{\text {twisted }} & \sim \int d^{6} y \sqrt{|\operatorname{det} g|}\left(1+\left(\mathcal{H}^{(3)}\right)^{2}+\ldots\right)-\int d^{6} y \sqrt{|\operatorname{det} g|}= \\
& =\int d^{6} y \sqrt{|\operatorname{det} g|}\left(\mathcal{H}^{(3)}\right)^{2}+\ldots
\end{aligned}
$$

Here $\mathcal{H}^{(3)}$ is the field strength of the self-dual 2-form potential of the NS5Abrane, and the self-duality condition is impossed at the level of the equations of motion. The metric is restricted to the position of the orientifold fixed plane.

\section{The non-BPS D2-brane of IIB/(-1) ${ }^{F_{L}} I_{4}$}

Performing now a T-duality transformation of the D1-brane solution along the $y^{4}$ direction we obtain a D2-brane solution of IIB $/(-1)^{F_{L}} I_{4}$. This brane is located at one of the fixed points of the orbifold, with its worldvolume extending along the non-compact spacetime. Taking the non-BPS D1-brane in the compactified orbifold at the critical radii, where it is possible to construct periodic infinite arrays of strings, and applying the T-duality rules we find:

$$
\begin{aligned}
& d s_{D 2}^{2}=\left(1-\frac{\kappa_{6} T_{2}}{2 \pi^{2} \Omega_{2}} \frac{1}{|y|}+\ldots\right)\left(-d t^{2}+d \sigma_{1}^{2}+d \sigma_{2}^{2}\right)+ \\
& \quad+\left(1+\frac{\kappa_{6} T_{2}}{2 \pi^{2} \Omega_{2}} \frac{1}{|y|}+\ldots\right)\left(\delta_{m n} d y^{m} d y^{n}+\delta_{i j} d x^{i} d x^{j}\right) \\
& \quad m, n=1, \ldots 3 ; i, j=1, \ldots 4 \\
& e^{\phi}=1+\frac{1}{2} \frac{\kappa_{6} T_{2}}{2 \pi^{2} \Omega_{2}} \frac{1}{|y|}+\ldots \\
& C_{0 \sigma_{1} \sigma_{2}}^{(3)}=-\frac{\kappa_{6} Q_{2}}{\sqrt{2} \Omega_{2}} \frac{1}{|y|}+\ldots .
\end{aligned}
$$

Here $T_{2}$ is the tension of the brane, $Q_{2}$ its charge and $\Omega_{2}$ the area of the unit 2 -sphere surrounding the 2-brane. 
The same analysis of the previous section gives the following form for the solution in the uncompactified case:

$$
\begin{aligned}
& d s_{D 2}^{2}=\left(1-\frac{1}{5} \frac{\kappa_{10} T_{2}}{\Omega_{6}} \frac{1}{|x|^{5}}+\ldots\right)\left(-d t^{2}+d \sigma_{1}^{2}+d \sigma_{2}^{2}\right)+ \\
& +\left(1+\frac{1}{5} \frac{\kappa_{10} T_{2}}{\Omega_{6}} \frac{1}{|x|^{5}}+\ldots\right)\left(\delta_{m n} d y^{m} d y^{n}+\delta_{i j} d x^{i} d x^{j}\right) \\
& m, n=1, \ldots 3 ; i, j=1, \ldots 4 \\
& e^{\phi}=1+\frac{1}{10} \frac{\kappa_{10} T_{2}}{\Omega_{6}} \frac{1}{|x|^{5}}+\ldots \\
& C_{0 \sigma_{1} \sigma_{2}}^{(3)}=-\frac{\kappa_{6} Q_{2}}{\sqrt{2} \Omega_{2}} \frac{1}{|y|}+\ldots
\end{aligned}
$$

Now $\Omega_{6}$ is the area of the unit 6 -sphere surrounding the membrane.

This brane is electrically charged with respect to the 3 -form potential of the NS5B-O5 system, or equivalently, magnetically charged with respect to its vector potential. Therefore, it solves the equations of motion derived from $S_{\text {untwisted }}+S_{\text {twisted }}$, with:

$$
\begin{aligned}
S_{\text {twisted }} & \sim \int d^{6} y \sqrt{|\operatorname{det} g|}\left(1+\left(\tilde{\mathcal{F}}^{(4)}\right)^{2}+\ldots\right)-\int d^{6} y \sqrt{|\operatorname{det} g|}= \\
& =\int d^{6} y \sqrt{|\operatorname{det} g|}\left(\tilde{\mathcal{F}}^{(4)}\right)^{2}+\ldots,
\end{aligned}
$$

where we have dualized the vector field of the NS5B-brane onto a 3-form potential with field strength $\tilde{\mathcal{F}}^{(4)}$, and the metric is restricted to the position of the orientifold fixed plane.

\section{The non-BPS M1-brane of M-theory $/ \Omega_{\rho} I_{5}$}

Oxidizing the D1-brane solution of the Type IIA theory on the orbifold we can obtain the expression for a stable M1-brane solution of M-theory orientifolded by $\Omega_{\rho} I_{5}$. In the compact case we obtain:

$$
d \hat{s}_{M 1}^{2}=\left(1-\frac{5}{6} \frac{\kappa_{6} T_{1}}{2 \pi^{2} \Omega_{3}} \frac{1}{|y|^{2}}+\ldots\right)\left(-d t^{2}+d \sigma^{2}\right)+
$$




$$
\begin{aligned}
& +\left(1+\frac{1}{6} \frac{\kappa_{6} T_{1}}{2 \pi^{2} \Omega_{3}} \frac{1}{|y|^{2}}+\ldots\right)\left(\delta_{m n} d y^{m} d y^{n}+\delta_{i j} d x^{i} d x^{j}\right)+ \\
& +\left(1+\frac{2}{3} \frac{\kappa_{6} T_{1}}{2 \pi^{2} \Omega_{3}} \frac{1}{|y|^{2}}+\ldots\right) d z^{2} \\
& m, n=1, \ldots 4 ; i, j=1, \ldots 4, \\
\hat{C}_{0 \sigma}^{(2)}= & \frac{1}{2} \frac{\kappa_{6} Q_{1}}{\sqrt{2} \Omega_{3}} \frac{1}{|y|^{2}}+\ldots
\end{aligned}
$$

This solution has an $S O(1,1) \times S O(4) \times S O(4) \times U(1)$ symmetry, i.e. it corresponds to an asymmetric orbifold. This is in agreement with the results of [22, 23], which show that the orbifold corresponding to M-theory on $T^{5} / \Omega_{\rho} I_{5}$ has to be asymmetric so that the twisted sector cancels the gravitational anomalies involved in the construction. We see that asymptotically, i.e. in the region where the dilaton of the Type IIA theory is of order 1 , the orbifold regains isotropy, also in agreement with [22, 23].

Uplifting the D1-brane solution corresponding to Type IIA on the uncompactified orbifold we find an M1-brane on $R^{1,5} \times\left(R^{4} \times S^{1}\right) / \Omega_{\rho} I_{5}$ :

$$
\begin{aligned}
d \hat{s}_{M 1}^{2} & =\left(1-\frac{5}{18} \frac{\kappa_{10} T_{1}}{\Omega_{7}} \frac{1}{|x|^{6}}+\ldots\right)\left(-d t^{2}+d \sigma^{2}\right)+ \\
& +\left(1+\frac{1}{18} \frac{\kappa_{10} T_{1}}{\Omega_{7}} \frac{1}{|x|^{6}}+\ldots\right)\left(\delta_{m n} d y^{m} d y^{n}+\delta_{i j} d x^{i} d x^{j}\right)+ \\
& +\left(1+\frac{2}{9} \frac{\kappa_{10} T_{1}}{\Omega_{7}} \frac{1}{|x|^{6}}+\ldots\right) d z^{2} ; \\
& m, n=1, \ldots 4 ; i, j=1, \ldots 4 \\
\hat{C}_{0 \sigma}^{(2)}= & \frac{1}{2} \frac{\kappa_{6} Q_{1}}{\sqrt{2} \Omega_{3}} \frac{1}{|y|^{2}}+\ldots
\end{aligned}
$$

As we did in the previous sections we can interpret this solution as an M1brane located at the origin of the $z$-circle, and find the expression for the corresponding solution in the completely uncompactified case:

$$
d \hat{s}_{M 1}^{2}=\left(1-\frac{5}{21} \frac{\hat{\kappa}_{11} \hat{T}_{1}}{\Omega_{8}} \frac{1}{|x|^{7}}+\ldots\right)\left(-d t^{2}+d \sigma^{2}\right)+
$$




$$
\begin{aligned}
& +\left(1+\frac{1}{21} \frac{\hat{\kappa}_{11} \hat{T}_{1}}{\Omega_{8}} \frac{1}{|x|^{7}}+\ldots\right)\left(\delta_{m n} d y^{m} d y^{n}+\delta_{i j} d x^{i} d x^{j}\right)+ \\
& +\left(1+\frac{4}{21} \frac{\hat{\kappa}_{11} \hat{T}_{1}}{\Omega_{8}} \frac{1}{|x|^{7}}+\ldots\right) d z^{2} .
\end{aligned}
$$

Here $\Omega_{8}$ is the area of the unit 8-sphere surrounding the string, and we have used $\hat{\kappa}_{11}=\kappa_{10}\left(2 \pi R_{z}\right)^{1 / 2}, \hat{T}_{1}=T_{1} \hat{\kappa}_{11} / \kappa_{10}$. $\hat{C}^{(2)}$ remains the same since it lives in the twisted sector.

Finally, the contribution to the supergravity action from the twisted sector is proportional to the worldvolume action describing an M5-O5 system, which in quadratic approximation reads:

$$
\hat{S}_{\text {twisted }} \sim \int d^{6} \hat{y} \sqrt{|\operatorname{det} \hat{g}|}\left(\hat{\mathcal{H}}^{(3)}\right)^{2}+\ldots
$$

Here $\hat{\mathcal{H}}^{(3)}$ is the field strength associated to the self-dual 2-form potential of the M5-brane worldvolume, with the self-duality condition impossed at the level of the equations of motion, and the metric is restricted to the position of the orientifold fixed plane.

\section{Acknowledgements}

It is a pleasure to thank Laurent Houart for very interesting discussions, and Eduardo Eyras for pointing out some mistakes in a previous version of this paper.

\section{References}

[1] A. Sen, Non-BPS States and Branes in String Theory, hep-th/9904207.

[2] A. Lerda and R. Russo, Stable Non-BPS States in String Theory: a Pedagogical Review, hep-th/9905006.

[3] J.H. Schwarz, TASI Lectures on Non-BPS D-Brane Systems, hep-th/9908144.

[4] O. Bergman and M. Gaberdiel, NonBPS Dirichlet Branes, hep-th/9908126.

[5] A. Sen, Strong Coupling Dynamics of Branes from M-theory, J. High Energy Phys. 10 (1997) 002, hep-th/9708002.

[6] R. Emparan, Black diholes, hep-th/9906160. 
[7] D. Youm, Delocalized Supergravity Solutions for Brane/Anti-brane Systems and their Bound States, hep-th/9908182.

[8] A. Chattaraputi, R. Emparan and A. Taormina, Composite diholes and intersecting brane-antibrane configurations in string/M-theory, hep-th/9911007.

[9] S. Mukherji, On the heterotic dipole at strong coupling, hep-th/9903012.

[10] B. Janssen and S. Mukherji, Kaluza-Klein dipoles, brane/anti-brane pairs and instabilities, hep-th/9905153.

[11] J.A. Harvey, P. Hor̆ava and P. Kraus, D-Sphalerons and the Topology of String Configuration Space, hep-th/0001143.

[12] E. Eyras and S. Panda, The Spacetime Life of a non-BPS D-particle, hep-th/0003033.

[13] A. Sen, Stable Non-BPS Bound States of BPS D-branes, J. High Energy Phys. 08 (1998) 010, hep-th/9805019.

[14] O. Bergman and M.R. Gaberdiel, Stable non-BPS D-particles, Phys. Lett. B441 (1998) 133, hep-th/9806155.

[15] A. Sen, Stable Non-BPS States in String Theory, J. High Energy Phys. 06 (1998) 007, hep-th/9803194.

[16] M.R. Gaberdiel and A. Sen, Non-supersymmetric D-Brane Configurations with Bose-Fermi Degenerate Open String Spectrum, J. High Energy Phys. 11 (1999) 008, hep-th/9908060.

[17] M. Mihailescu, K. Oh and R. Tatar, Non-BPS Branes on a Calabi-Yau Threefold and Bose-Fermi Degeneracy, hep-th/9910249.

[18] N.D. Lambert and I. Sachs, Non-abelian Field Theory of Stable non-BPS Branes, hep-th/0002061.

[19] A. Sen, Duality and Orbifolds, Nucl. Phys. B474 (1996) 361, hep-th/9604070.

[20] D. Kutasov, Orbifolds and Solitons, Phys. Lett. B383 (1996) 48, hep-th/9512145.

[21] J. Majumder and A. Sen, 'Blowing up' D-branes on Non-supersymmetric cycles, J. High Energy Phys. 09 (1999) 004, hep-th/9906109. 
[22] K. Dasgupta and S. Mukhi, Orbifolds of M-theory, Nucl. Phys. B465 (1996) 399, hep-th/9512196.

[23] E. Witten, Five-branes and M-theory on an Orbifold, Nucl. Phys. B463 (1996) 383, hep-th/9512219.

[24] P. Hor̆ava and E. Witten, Heterotic and Type I String Dynamics from Eleven Dimensions, Nucl. Phys. B460 (1996) 506, hep-th/9510209.

[25] P. Di Vecchia, M.L. Frau, A. Lerda, I. Pesando, R. Russo and S. Sciuto, Classical p-Branes from Boundary State, Nucl. Phys. B507 (1997) 259, hep-th/9707068.

[26] R. Argurio, Brane Physics in M-theory, PhD thesis, hep-th/9807171. 